\title{
PERIODIC SOLUTIONS OF SINGULAR SYSTEMS WITHOUT THE STRONG FORCE CONDITION
}

\author{
DANIEL FRANCO AND PEDRO J. TORRES \\ (Communicated by Carmen C. Chicone)
}

\begin{abstract}
We present sufficient conditions for the existence of at least a noncollision periodic solution for singular systems under weak force conditions. We deal with two different types of systems. First, we assume that the system is generated by a potential, and then we consider systems without such hypothesis. In both cases we use the same technique based on Schauder fixed point theorem. Recent results in the literature are significantly improved.
\end{abstract}

\section{INTRODUCTION}

The main purpose of this paper is to study

$$
\ddot{x}(t)+\nabla_{x} V(t, x(t))=f(t)
$$

where $V \in C^{1}\left(\mathbb{R} \times \mathbb{R}^{N} \backslash\{0\}, \mathbb{R}\right)$ is a singular potential and $f \in L^{1}\left(\mathbb{R} / T \mathbb{Z}, \mathbb{R}^{N}\right)$. We shall assume that $V$ and $f$ are $T$-periodic in $t$. In such a situation it is natural to look for $T$-periodic non-collision solutions of (1.1). Here, as usual, by a $T$-periodic non-collision solution, we mean a function $u \in H^{1}\left(\mathbb{R} / T \mathbb{Z}, \mathbb{R}^{N}\right)$ solving (1.1) and such that $u(t) \neq 0$ for all $t$. If necessary, see [4] for the definitions of the above functional spaces.

We shall assume that (1.1) has a singularity of repulsive type in 0 , i.e.

$$
\lim _{|x| \rightarrow 0} V(t, x)=+\infty \quad \text { uniformly in } t .
$$

Singular potentials appear when gravitational or electromagnetic forces are considered. The problem of finding periodic solutions for (1.1) in the situation described above has generated much interest in the last decades (see [3] and the extensive bibliography therein). The variational arguments have been clearly the most used tools, although other techniques have been used (see for example [5]). In relation with the variational approach, a common hypothesis is the unboundedness of the action functional near the singularity to guarantee that its critical points have no collisions with the singularity. This condition has been known at least since the times of Poincaré [11]. Nowadays, it is known as the "strong force condition" in a

Received by the editors August 17, 2006.

2000 Mathematics Subject Classification. Primary 37J45, 34C25, 34B16.

The first author was supported by D.G.I. MTM2004-06652-C03-03, Ministerio de Educación y Ciencia, Spain.

The second author was supported by D.G.I. MTM2005-03483, Ministerio de Educación y Ciencia, Spain. 
term first used by Gordon in [6]. Roughly, it establishes a maximum rate of growth of the potential near the singularity. For example, if

$$
V(t, x)=\frac{1}{|x|^{\alpha+1}}
$$

the strong force condition holds for $\alpha \geq 1$. Therefore, important cases such as Newtonian-type potentials do not satisfy that condition. Even in the scalar case, a weak singularity may lead to collisions, as it is shown in the classical paper [8]. The behavior of solutions with collisions is a difficult topic not very well understood up to now. For attractive singularities, it is closely related with the regularization techniques frequently employed in Celestial Mechanics. In the weak repulsive case, an impact model for the continuation of the solutions after a collision was proposed in [12]. We refer the reader to $[1,2,13]$ and the references therein for further literature on problems with weak forces.

In Section 2, we shall consider the potential

$$
V(t, x)=a(t) \frac{|x|^{2}}{2}-g\left(t, \frac{|x|^{2}}{2}\right)
$$

with a $T$-periodic dependence on $t$ and such that $g$ presents a singularity of the repulsive type. Our viewpoint sheds some new light on problems with weak force potentials.

In order to prove our results we shall adapt a technique recently presented for a nonlinear Hill's equation [14], which relies on Schauder's fixed point theorem. There are examples in the literature of techniques that work well for the one dimensional case without assuming the strong force condition, but one needs to consider it to deal with higher dimensional systems (see Remark 1 in [17]). As we will see, that is not our case.

The last section of the paper will be dedicated to the study of systems with singularities but not necessarily those generated by a potential. This section was motivated by the recent paper [9]. There the authors use a Leray-Schauder alternative and a fixed point theorem in cones to guarantee the existence of twin positive periodic solutions for the system

$$
\ddot{x}(t)+a(t) x(t)=F(x(t))
$$

where $F$ can be expressed as a sum of two positive functions satisfying certain monotone conditions which prevent us from considering system (1.1) with $V$ as in (1.2). As an example they show that their results guarantee the existence of solution for the system

$$
\begin{aligned}
& \ddot{x}(t)+a_{1}(t) x(t)=\sqrt{\left(x^{2}+y^{2}\right)^{-\alpha}}+\mu \sqrt{\left(x^{2}+y^{2}\right)^{\beta}}, \\
& \ddot{y}(t)+a_{2}(t) y(t)=\sqrt{\left(x^{2}+y^{2}\right)^{-\alpha}}+\mu \sqrt{\left(x^{2}+y^{2}\right)^{\beta}}
\end{aligned}
$$

when $\alpha>0$ and $\beta \in[0,1)$ for every positive constant $\mu$. We shall present results improving the above result, since we shall show that when the singularity is weak enough that $\mu$ can be zero or even negative.

As we will see, one of the advantages of using the Schauder fixed point theorem instead of the Leray-Schauder alternative relies on the fact that we do not need to make a technical truncation to get compactness because we can work in a set where the singularity is excluded. 


\section{EXISTENCE RESUlts I}

When $V$ is the potential defined in (1.2), system (1.1) can be written as

$$
\ddot{x}+a(t) x=D_{2} g\left(t, \frac{|x|^{2}}{2}\right) x+f(t),
$$

where $D_{2} g$ denotes the partial derivative of $g$ with respect to the second variable.

Let us comment on the particular case $a(t) \equiv k^{2}$. It is well known (see, for example, [5]) that for $k \in\left(0, \frac{\pi}{T}\right]$ the Green's function of the periodic problem

$$
G(t, s)= \begin{cases}\frac{\sin k(t-s)+\sin k(T-t+s)}{2 k(1-\cos k T)}, & 0 \leq s \leq t \leq T, \\ \frac{\sin k(s-t)+\sin k(T-s+t)}{2 k(1-\cos k T)}, & 0 \leq t \leq s \leq T,\end{cases}
$$

is non-negative.

In the general case, we will assume the standing assumption:

(A) The Hill's equation $x^{\prime \prime}+a(t) x=0$ is non-resonant (i.e. the unique periodic solution is the trivial one) and the corresponding Green's function $G(t, s)$ is non-negative for every $(t, s) \in[0, T] \times[0, T]$.

For a non-constant $a(t)$, there is no explicit expression of the Green's function, but there is an $L^{p}$-criterion for the positiveness of the Green's function proved in [15] (the proof relies on an anti-maximum principle from [16]) which is becoming standard in related literature (see for instance $[7,9,14]$ ).

As usual we denote by $f_{1}, \ldots, f_{N}$ the components of a given function $f$ with values on $\mathbb{R}^{N}$. For $f \in L^{1}\left(\mathbb{R} / T \mathbb{Z}, \mathbb{R}^{N}\right)$ we define the function $\gamma: \mathbb{R} \rightarrow \mathbb{R}^{N}$ by

$$
\gamma_{i}(t)=\int_{0}^{T} G(t, s) f_{i}(s) d s, \quad i=1, \ldots, N,
$$

which is just the unique $T$-periodic solution of

$$
\ddot{x}+a(t) x=f(t) .
$$

Let us denote

$$
\gamma_{*}=\min _{i, t} \gamma_{i}(t) \quad \text { and } \quad \gamma^{*}=\max _{i, t} \gamma_{i}(t) .
$$

Theorem 2.1. Let us assume (A) holds, $\gamma_{*}>0$, and that the potential $V$ satisfies

(H1) $D_{2} g(t, s) \geq 0$,

(H2) $D_{2} g(t, s) \leq \frac{c}{s^{\alpha}}$ with $\alpha, c>0$,

for all $t \in \mathbb{R}, s>0$. Then, there exists a T-periodic solution $x$ of (1.1) such that $x_{i} \geq \gamma_{*}$ for $i=1, \ldots, N$.

Proof. Consider the closed, convex and bounded set

$$
K=\left\{x \in C\left(\mathbb{R} / T \mathbb{Z}, \mathbb{R}^{N}\right): r \leq x_{i}(t) \leq R\right\}
$$

where $r, R>0$ are chosen later.

We are going to show that there exists a $T$-periodic solution belonging to $K$. Clearly, finding such a solution is equivalent to finding a fixed point for the operator $\mathcal{T}: K \subset C\left(\mathbb{R} / T \mathbb{Z}, \mathbb{R}^{N}\right) \rightarrow C\left(\mathbb{R} / T \mathbb{Z}, \mathbb{R}^{N}\right)$ defined by

$$
\mathcal{T} x(t)=\int_{0}^{T} G(t, s)\left[D_{2} g\left(t, \frac{|x(s)|^{2}}{2}\right) x(s)+f(s)\right] d s .
$$


Note that since $\mathcal{T}$ is defined on $K$ we avoid the singularity and standard reasoning shows that $\mathcal{T}$ is compact (see [10] for more details).

Next, we shall prove that $\mathcal{T}(K) \subset K$. Therefore, as a consequence of the Schauder fixed point theorem, the existence of the $T$-periodic solution is guaranteed.

Taking $r=\gamma_{*}$, we have for each $i \in\{1, \ldots, N\}$ and $x \in K$

$$
(\mathcal{T} x)_{i}(t)=\int_{0}^{T} G(t, s)\left[D_{2} g\left(t, \frac{|x(s)|^{2}}{2}\right) x_{i}(s)+f_{i}(s)\right] d s \geq \min _{t} \gamma_{i}(t) \geq r .
$$

On the other hand, for each $i \in\{1, \ldots, N\}$ we have

$$
\begin{aligned}
(\mathcal{T} x)_{i}(t) & \leq \int_{0}^{T} G(t, s) D_{2} g\left(t, \frac{|x|^{2}}{2}\right) x_{i} d s+\gamma^{*} \\
& \leq \int_{0}^{T} G(t, s) \frac{c 2^{\alpha}}{\left(x_{i}^{2}\right)^{\alpha}} x_{i} d s+\gamma^{*} \\
& =c 2^{\alpha} \int_{0}^{T} G(t, s) x_{i}^{1-2 \alpha} d s+\gamma^{*} .
\end{aligned}
$$

Now, we consider two cases.

- Case $\alpha>\frac{1}{2}$ : Since for $x \in K$

$$
c 2^{\alpha} \int_{0}^{T} G(t, s) x_{i}^{1-2 \alpha} d s \leq c 2^{\alpha} r^{1-2 \alpha} \int_{0}^{T} G(t, s) d s,
$$

we deduce that it is sufficient to choose $R$ such that

$$
c 2^{\alpha} r^{1-2 \alpha} \int_{0}^{T} G(t, s) d s+\gamma^{*}<R .
$$

- Case $0<\alpha \leq \frac{1}{2}$ : For $x \in K$ we have

$$
c 2^{\alpha} \int_{0}^{T} G(t, s) x_{i}^{1-2 \alpha} d s \leq c 2^{\alpha} R^{1-2 \alpha} \int_{0}^{T} G(t, s) d s .
$$

Therefore, we are done because for $R$ big enough one has

$$
c 2^{\alpha} R^{1-2 \alpha} \int_{0}^{T} G(t, s) d s+\gamma^{*}<R
$$

Remark 2.2. Note that the constant $c>0$ helps us to locate the solution in the following sense: smaller is the constant $c>0$, and smaller is the set $K$ to which the solution belongs.

If $\alpha$ is small enough we can improve the condition on the function $\gamma$ in Theorem 2.1 .

Theorem 2.3. Let us assume that there exists $i \in\{1, \ldots, N\}$ such that $\min _{t} \gamma_{i}(t)>$ 0 . Besides, let us assume that the conditions (A), (H1) and (H2) hold with $0<$ $\alpha \leq \frac{1}{2}$. Then, there exists a T-periodic solution of (1.1). 
Proof. Let $\Lambda=\left\{i: \min _{t} \gamma_{i}(t)>0\right\} \neq \emptyset$ and consider the closed, convex and bounded set

$$
\widehat{K}=\left\{x \in C\left(\mathbb{R} / T \mathbb{Z}, \mathbb{R}^{N}\right): r \leq x_{i}(t) \leq R \text { if } i \in \Lambda ;\left|x_{i}\right| \leq R \text { in the other case }\right\}
$$

where $r, R>0$ are chosen later. Again $\mathcal{T}: \widehat{K} \rightarrow C\left(\mathbb{R} / T \mathbb{Z}, \mathbb{R}^{N}\right)$, defined by $(2.1)$, is a compact operator.

Following the proof of Theorem 2.1 we easily see that

$$
r \leq(\mathcal{T} x)_{i}(t) \leq R
$$

for $i \in \Lambda$. Now take $i \in\{1, \ldots, N\} \backslash \Lambda$

$$
\begin{aligned}
\left|(\mathcal{T} x)_{i}(t)\right| & \leq\left|\int_{0}^{T} G(t, s)\left[D_{2} g\left(t, \frac{|x|^{2}}{2}\right) x_{i}+f_{i}(s)\right] d s\right| \\
& \leq \int_{0}^{T} G(t, s) \frac{c 2^{\alpha}\left|x_{i}\right|}{\left(x_{i}^{2}\right)^{\alpha}} d s+\left|\gamma_{i}(t)\right| \\
& \leq c 2^{\alpha} \int_{0}^{T} G(t, s)\left|x_{i}\right|^{1-2 \alpha} d s+\max _{t}\left|\gamma_{i}(t)\right| \\
& \leq \frac{c 2^{\alpha}}{k^{2}} R^{1-2 \alpha}+\max _{t}\left|\gamma_{i}(t)\right| .
\end{aligned}
$$

Therefore, choosing $R$ large enough we have $\mathcal{T}(\widehat{K}) \subset \widehat{K}$ and there exists a periodic solution.

Remark 2.4. Besides the lower bound given in the statement of Theorem 2.1, from the proof it is easy to deduce an upper bound for the solution depending on the value of $\alpha$. For example, if $\alpha>\frac{1}{2}$ and $a \equiv k^{2}$ we get that

$$
x_{i} \leq \frac{c 2^{\alpha}}{k^{2}} \gamma_{*}^{1-2 \alpha}+\gamma^{*} .
$$

On the other hand, it is also possible to get some estimates for the periodic solution in Theorem 2.3. For example, for $i \in \Lambda$ we have

$$
x_{i} \geq \min _{i \in \Lambda, t} \gamma_{i}(t)
$$

\section{EXISTENCE RESUlts II}

In this section we study the system

$$
\ddot{x}(t)+a(t) x(t)=F(t, x(t))
$$

with $F$ a $T$-periodic function in the first variable and $a \in L^{1}\left(\mathbb{R} / T \mathbb{Z}, \mathbb{R}^{N}\right)$ such that each component $a_{i}$ satisfies the standing hypothesis (A). In this situation $G(t, s)$ is a vectorial Green's function whose components are the Green's functions of each coefficient $a_{i}$, all of them non-negative by assumption.

Our main idea is to obtain sufficient conditions for the existence of at least a periodic positive solution for

$$
\begin{aligned}
& \ddot{x}(t)+a_{1}(t) x(t)=\sqrt{\left(x^{2}+y^{2}\right)^{-\alpha}}+\mu \sqrt{\left(x^{2}+y^{2}\right)^{\beta}}, \\
& \ddot{y}(t)+a_{2}(t) y(t)=\sqrt{\left(x^{2}+y^{2}\right)^{-\alpha}}+\mu \sqrt{\left(x^{2}+y^{2}\right)^{\beta}}
\end{aligned}
$$

when $\alpha>0, \beta \in[0,1)$ and $\mu \in \mathbb{R}$. 
Now, for $f \in L^{1}\left(\mathbb{R} / T \mathbb{Z}, \mathbb{R}^{N}\right)$ we define $\gamma: \mathbb{R} \rightarrow \mathbb{R}^{N}$ by

$$
\gamma_{i}(t)=\int_{0}^{T} G_{i}(t, s) f_{i}(s) d s, \quad i=1, \ldots, N,
$$

and as before we denote $\gamma_{*}=\min _{i, t} \gamma_{i}(t)$ and $\gamma^{*}=\max _{i, t} \gamma_{i}(t)$.

Theorem 3.1. Suppose that there exist $\alpha>0, \beta \in[0,1), b \in L^{1}\left(\mathbb{R} / T \mathbb{Z}, \mathbb{R}^{+}\right)$and $f \in L^{1}\left(\mathbb{R} / T \mathbb{Z}, \mathbb{R}^{N}\right)$ such that each component $F_{i}$ of $F$ satisfies

$$
f_{i}(t)|x|^{\beta} \leq F_{i}(t, x) \leq \frac{b(t)}{|x|^{\alpha}}+f_{i}(t)|x|^{\beta} .
$$

If $f$ is such that $\gamma_{*}>0$, then there exists a T-periodic solution of (3.1).

Proof. Consider $K=\left\{x \in C\left(\mathbb{R} / T \mathbb{Z}, \mathbb{R}^{N}\right): r \leq x_{i}(t) \leq R\right\}$. We have to prove that $\widehat{\mathcal{T}}(K) \subset K$ where now

$$
\widehat{\mathcal{T}} x(t)=\int_{0}^{T} G(t, s) F(t, x(s)) d s .
$$

For each $i \in\{1, \ldots, N\}$, one obtains

$$
(\widehat{\mathcal{T}} x)_{i}(t) \geq \int_{0}^{T} G_{i}(t, s) f_{i}(s) r^{\beta} d s \geq r^{\beta} \gamma_{*}
$$

and

$$
(\widehat{\mathcal{T}} x)_{i}(t) \leq \int_{0}^{T} G_{i}(t, s)\left[f_{i}(s)(\sqrt{N} R)^{\beta}+\frac{b(s)}{r^{\alpha}}\right] d s \leq \gamma^{*}(\sqrt{N} R)^{\beta}+\frac{B_{i}}{r^{\alpha}}
$$

where $B_{i}=\int_{0}^{T} G_{i}(t, s) b(s) d s$.

We have finished, since $\beta \in[0,1)$ guaranties that the following inequalities hold for $r$ small enough and $R$ big enough:

$$
r^{\beta} \gamma_{*}>r, \quad \gamma^{*}(\sqrt{N} R)^{\beta}+\frac{B_{i}}{r^{\alpha}}<R .
$$

Corollary 3.2. System (3.2) has a positive periodic solution for every $\mu \in(0, \infty)$.

The following result assures us that (3.2) still has a positive solution when $\mu=0$ if $\alpha$ is sufficiently small.

Theorem 3.3. Suppose that there exist $\alpha \in(0,1), \beta \in[0,1), f \in L^{1}\left(\mathbb{R} / T \mathbb{Z}, \mathbb{R}^{N}\right)$ and $b, \tilde{b} \in L^{1}\left(\mathbb{R} / T \mathbb{Z}, \mathbb{R}^{+}\right)$such that each component $F_{i}$ of $F$ satisfies

$$
\frac{\tilde{b}(t)}{|x|^{\alpha}}+f_{i}(t)|x|^{\beta} \leq F_{i}(t, x) \leq \frac{b(t)}{|x|^{\alpha}}+f_{i}(t)|x|^{\beta} .
$$

If $f$ is such that $\gamma_{*}=0$, then there exists a T-periodic solution of (3.1).

Proof. Considering the same set $K$ as in the preceding result and denoting $\tilde{B}_{i}=$ $\int_{0}^{T} G_{i}(t, s) \tilde{b}(s) d s$ and $B_{i}=\int_{0}^{T} G_{i}(t, s) b(s) d s$, we obtain

$$
(\widehat{\mathcal{T}} x)_{i}(t) \geq \int_{0}^{T} G_{i}(t, s) \frac{\tilde{b}(s)}{|x(s)|^{\alpha}} d s \geq \frac{\tilde{B}_{i}}{(\sqrt{N} R)^{\alpha}}
$$

and

$$
(\widehat{\mathcal{T}} x)_{i}(t) \leq \int_{0}^{T} G_{i}(t, s)\left[f_{i}(s)|x(s)|^{\beta}+\frac{b(s)}{|x(s)|^{\alpha}}\right] d s \leq \gamma^{*}(\sqrt{N} R)^{\beta}+\frac{B_{i}}{r^{\alpha}}
$$


Thus, we need to fix $r<R$ such that

$$
\frac{\tilde{B}_{i}}{(\sqrt{N} R)^{\alpha}} \geq r, \quad \gamma^{*}(\sqrt{N} R)^{\beta}+\frac{B_{i}}{r^{\alpha}} \leq R
$$

for any $i=1, \ldots, N$.

Taking $R=1 / r$, it is sufficient to find $R>1$ such that

$$
\frac{\tilde{B}_{i}}{(\sqrt{N})^{\alpha}} R^{1-\alpha} \geq 1, \quad \gamma^{*}(\sqrt{N} R)^{\beta}+B_{i} R^{\alpha} \leq R,
$$

and these inequalities hold for $R$ big enough because $\alpha \in(0,1)$ and $\beta \in[0,1)$.

Our last result allows us to explore the existence of positive solutions for (3.2) when $\mu<0$, which is well known as a more difficult situation. We need to assume that $\beta=0$ and $\alpha \in(0,1)$ although we believe these hypotheses can be weakened. The proof would be almost the same as in Theorem 4 of [14], so we leave it to the reader.

Theorem 3.4. Suppose that there exist $\alpha \in(0,1), b, \tilde{b} \in L^{1}\left(\mathbb{R} / T \mathbb{Z}, \mathbb{R}^{+}\right)$and $f \in$ $L^{1}\left(\mathbb{R} / T \mathbb{Z}, \mathbb{R}^{N}\right)$ such that each component $F_{i}$ of $F$ satisfies

$$
\frac{\tilde{b}(t)}{|x|^{\alpha}}+f_{i}(t) \leq F_{i}(t, x) \leq \frac{b(t)}{|x|^{\alpha}}+f_{i}(t) .
$$

If $f$ is such that $\gamma^{*}<0$ and

$$
\gamma_{*} \geq\left[\frac{\tilde{B}}{(\sqrt{N} B)^{\alpha}} \alpha^{2}\right]^{\frac{1}{1-\alpha^{2}}}\left(1-\frac{1}{\alpha^{2}}\right)
$$

then there exists a T-periodic solution of (3.1).

Of course, we do not need all the components of $\gamma$ to be negative. We have chosen this situation for clarity and because it covers our model (3.2). However, choosing an adequate set $K$ and making straightforward variations of the technique one could consider

$$
\begin{aligned}
& \ddot{x}(t)+a_{1}(t) x(t)=\sqrt{\left(x^{2}+y^{2}\right)^{-\alpha}}+\mu_{1} \sqrt{\left(x^{2}+y^{2}\right)^{\beta}}, \\
& \ddot{y}(t)+a_{2}(t) y(t)=\sqrt{\left(x^{2}+y^{2}\right)^{-\alpha}}+\mu_{2} \sqrt{\left(x^{2}+y^{2}\right)^{\beta}},
\end{aligned}
$$

where $\mu_{1}, \mu_{2} \in \mathbb{R}$ can have a different sign. Of course, analogous results are obtained when the coefficients $\mu_{1}$ and $\mu_{2}$ are periodically dependent on time. The interested reader is invited to check out such direct generalizations.

As a final comment, let us emphasize that our standing hypothesis (A) (nonnegativeness) is weaker than the required condition in $[15,7,9]$ (positiveness of the Green's function) and allows, for instance, the critical case $a(t) \equiv \frac{\pi}{T}$.

\section{REFERENCES}

1. Adachi S., Non-collision periodic solutions of prescribed energy problem for a class of singular Hamiltonian systems, Topol. Methods Nonlinear Anal. 25 (2005) 275-296. MR2154429 (2006e:37113)

2. Adachi S., Tanaka K., Terui M., A remark on periodic solutions of singular Hamiltonian systems, NoDEA Nonlinear Differential Equations Appl. 12 (2005) 265-274. MR2186332 (2006j:37071) 
3. Ambrosetti A., Coti Zelati V. Periodic solutions of singular Lagrangian systems, Birkhäuser Boston, Boston, MA, 1993. MR1267225 (95b:58054)

4. Brézis, H., Analyse fonctionnelle, Masson, Paris, 1983. MR697382 (85a:46001)

5. Franco D., Webb J.R.L., Collisionless orbits of singular and non singular dynamical systems, Discrete Contin. Dyn. Syst. 15 (2006) 747-757. MR2220746 (2006m:34110)

6. Gordon W.B. Conservative dynamical systems involving strong forces, Trans. Amer. Math. Soc. 204 (1975) 113-135. MR0377983 (51:14152)

7. Jiang D., Chu J., Zhang M., Multiplicity of positive periodic solutions to superlinear repulsive singular equations, J. Differential Equations 211 (2005) no. 2, 282-302. MR2125544 (2005k:34087)

8. Lazer A.C., Solimini S., On periodic solutions of nonlinear differential equations with singularities, Proc. Amer. Math. Soc. 99 (1987), 109-114. MR866438 (87k:34064)

9. Lin X., Jiang D., O'Regan D., Agarwal R.P., Twin positive periodic solutions of second order singular differential systems, Topol. Methods Nonlinear Anal. 25 (2005) no. 2, 263-273. MR2154428 (2006d:34050)

10. Martin R.H., Nonlinear operators and differential equations in Banach spaces, John Wiley and Sons, New York, 1976. MR0492671 (58:11753)

11. Poincaré H., Sur les solutions périodiques et le priciple de moindre action, C.R. Math. Acad. Sci. Paris 22 (1896) 915-918.

12. Qian D., Torres P.J., Periodic motions of linear impact oscillators via the successor map, SIAM J. Math. Anal. 36 no. 6 (2005), 1707-1725. MR2178218 (2006g:34078)

13. Ramos M., Terracini S., Noncollision periodic solutions to some singular dynamical systems with very weak forces, J. Differential Equations 118 (1995) no. 1, 121-152. MR1329405 (96d:58115)

14. Torres P.J., Weak singularities may help periodic solutions to exist, J. Differential Equations 232 (2007) 277-284. MR2281196

15. Torres P.J., Existence of one-signed periodic solutions of some second-order differential equations via a Krasnoselskii fixed point theorem, J. Differential Equations 190 (2003) no. 2, 643-662. MR1970045 (2003k:34082)

16. Torres P.J., Zhang M., A monotone iterative scheme for a nonlinear second order equation based on a generalized anti-maximum principle. Math. Nachr. 251 (2003) 101-107. MR1960807 (2004a:34032)

17. Zhang M., Periodic solutions of damped differential systems with repulsive singular forces, Proc. Amer. Math. Soc. 127 (1999) 401-407. MR1637460 (99k:34092)

Departamento de Matemática Aplicada, uned, ETSi Industriales, C/ Juan del Rosal 12, 28040 MADRID, SPAIN

E-mail address: dfranco@ind.uned.es

Universidad de Granada, Departamento de Matemática Aplicada, 18071 Granada, SPAIN

E-mail address: ptorres@ugr.es 